\title{
INDUSTRIAL VISUAL INSPECTION OF LIME GRANULES BY NEURAL NETWORKS
}

\author{
P. Carvalho, N. Costa, B. Ribeiro, A. Dourado \\ CISUC - Centro de Informática e Sistemas da Universidade de Coimbra \\ Department of Engineering Informatics, Coimbra University, PT \\ fax: +35139701266 e-mail: carvalho@dei.uc.pt
}

\begin{abstract}
Lime granule quality inspection is an important task in the pulp and paper industry. In this paper a new method, build-up on a neural network and a path search method, is introduced for lime granule automatic visual inspection. Several correction steps to Landau's method are also introduced. () 1998 Elsevier Science Ltd. All rights reserved.
\end{abstract}

\section{KEYWORDS}

Automatic visual inspection; elliptical objects localisation; neural networks; pulp and paper industry

\section{INTRODUCTION}

Recycling is an economical as well as an environmental important issue for most industries. One of the recycling paths in the pulp and paper industry is used to recover bleaching liquor through a causticizing chemical reaction. The efficiency of this reaction is highly conditioned by the quality of the lime applied, namely by its granularity. This fact leads to the need for quality inspection of lime granules. Lime granules are more or less elliptical in shape and, therefore, their automatic visual inspection can be seen as an elliptical object localisation and measurement problem, traditionally solved with a model search procedure such as the Hough transform, least squares or least median squares fitting methods. These strategies exhibit, however, some important drawbacks: (i) low computational efficiency and (ii) low localisation performance whenever in the presence of highly irregular shapes as is the case for most lime granules. In this paper a new and robust method for elliptical object localisation is introduced and applied to the outlined inspection problem. Low level segmentation of lime granule images is not a major difficulty given their bi-modal histograms. We use a minimum cross-entropy principle (Brink and Pendock, 1996) to obtain the binary image. From this image all independent agglomerates of lime granules are identified and their contours grouped. For each cluster the set of contact points between distinct lime granules are then computed, which leads to the definition of a set of elementary arc segments (EAS). Finally the obtained $E A S$ which belong to the same elliptical object are aggregated together using a multilayer neural network, a path search algorithm and some geometrical-based decision criteria. Main results and conclusions are presented at the end of the paper.

\section{IDENTIFICATION OF EAS}

The method is based on the analysis of geometrical relationships between sets of globally convex arc segments, which we shall call $E A S$, obtained from the contours of each multi-connected region (cluster of lime granules). Random dispositions of lime granules may lead to several multi-connected regions formations in the image. Further, it is seen that one or more of these regions may be formed inside a cluster. 


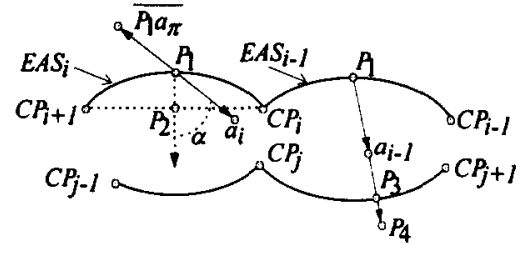

(a)

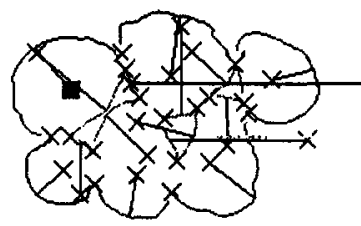

(b)

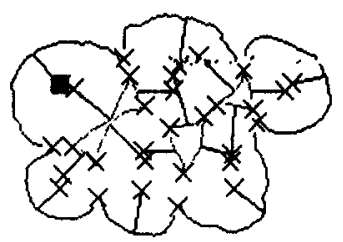

(c)

Fig. 1: a) Definitions of the points for the geometrical correction steps. b) Centre estimation using Landau's method; c) Centre estimation using Landau's method with correction steps.

However, it is known that all $E A S$ that belong to the same elliptical object must be part of the same cluster of lime granules. Therefore, the reconstruction analysis must be carried out for each independent cluster of lime granules. The identification of all agglomerates is performed using a contour grouping strategy based on the Green's discrete theorem (Carvalho et al., 1997). This grouping method assures a fundamental property for the aggregation method: all internal contours are stored with clockwise tracing direction while all external ones are stored with the inverse direction.

The reconstruction of lime granules is based on elementary arc aggregation. It is assumed that an $E A S$ is globally convex, therefore non-convex regions of the contour correspond to contact points between distinct lime granules. There are basically two types of strategies to compute these points: (i) polygonal approximation methods and (ii) curvature based methods. In this work we apply the incremental splitting algorithm introduced by (Esplid and Jonassen, 1991) which is computationally very efficient. This algorithm identifies extreme points in non-convex, as well as in convex contour regions. By applying the convexity criterion introduced in (Kim and Rosenfeld, 1991) it is seen that point $P_{i}$, obtained with the incremental splitting method, lies in a non-convex region if and only if $\left|\overline{P_{P} P_{m}}\right|<\Delta$ and $P_{m}$ lies outside the object $\left(P_{m}-\right.$ middle point of the line segment defined by the contour points $z$ position to the right and to the left to $P_{i} ; \Delta$ - user defined parameter). An $E A S$ is defined by a pair of adjacent contact points $\left\{C P_{i,} C P_{i+1}\right\}$ :

$$
E A S_{i}=\left\{C P_{i}, \ldots, P, \ldots, C P_{t+1}\right\} \in \text { Contour }
$$

Centres and radius of circular arc segments are quite straightforward to estimate using Landau's method (Landau, 1987). However, it is verified that this algorithm fails whenever (i) the arcs are small in size and (ii) the arcs are irregular, as is the case of most $E A S$ of lime granules. To overcome these problems a set of geometrical correction steps to the estimated radius and centres obtained with the Landau's method are introduced ( $R$ - region of support; $P_{1}$ - middle point of the $E A S ; P_{2}$ - middle point of $\overline{C P_{k} C P_{k+1}}$ ):

1. The angle formed by the vectors $\overrightarrow{P_{1} a}$ and $\vec{P}_{1} P_{2}$ is theoretically $0^{\circ}$. Given the irregular shapes of $E A S$, angles smaller than $\alpha$ (we take $\alpha=30^{\circ}$ ) are allowed, else the estimated centre $a$ is rotated $-\alpha$ degrees with respect to point $P_{1}$ (see Fig. la).

2. The identified centre $a$ of an $E A S$ must lie inside the region $R$ it belongs to, therefore: (i) obtain line segments $\overline{P_{1} a}$ and $\overline{P_{1} a_{\pi}}$ (see Fig. 1 a); (ii) if $\left|\overline{P_{1} a} \cap R\right| \overline{P_{1} a_{\pi}} \cap R \mid$ then rotate $a$ as in 1. with $\alpha=\pi$; (iii) if centre $a$ lies outside $R$ then compute point $P_{3}$ (see Fig. 1 a) and take $a=0.5\left(P_{1}+P_{3}\right)$.

3. For smooth arc segments it is seen that the modulus of the diameter is twice the modulus of the radius with the same direction. This property enables the correction of abnormal identified modulus values. Namely (see Fig. 1 a), if $\left(\left|\overline{P_{1} a}\right|<\delta\right.$ or $P_{4} \notin R$ and $\left.\left|\overline{P_{3} a}\right|<(1-\delta)\left|\overline{P_{1} a}\right|\right)$ then apply $a=0.5\left(P_{1}+P_{3}\right)$.

In Fig. Ic some results obtained with Landau's method and with the described correction steps are shown.

\section{THE LOCALISATION METHOD}

The identification of elliptical regions is performed upon a path search algorithm whose primary inputs are a set of membership coefficients obtained from a neural network. Several results can be seen in Fig. 2 . The neural network's inputs are derived from a set of geometrical measures, and its output reflects the possibility degree of two distinct $E A S$ belonging to the same object. The structure employed for the multilayer feedforward neural network is formed by 6 input, 9 hidden and 1 output neuron, all with a sigmoid type activation function. The network was trained using the back-propagation learning algorithm in a modified form including adaptive learning rate (Jacobs, 1988). The training set was composed by 841 


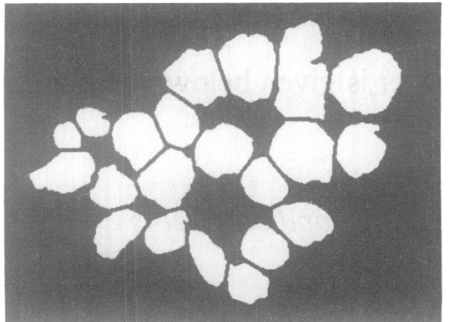

(a)

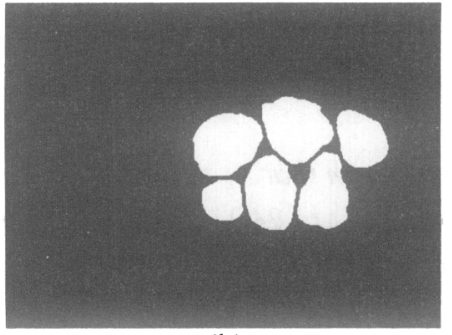

(b)

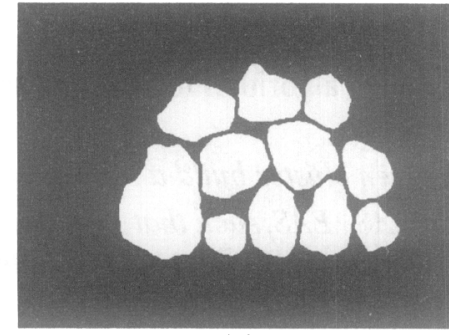

(c)

Fig. 2: Lime granule localisation results obtained with the described method.

distinct $E A S$. For training purposes, it is considered that if two $E A S$ belong to the same object, then the neural network's output is 0.9 , else the output is 0.1 . A set of geometrical-based similarity measures are employed as the network's inputs:

Proximity measure: Let $E A S_{i}$ and $E A S_{q}$ be two aggregation candidates, their aggregation distance is given by $D^{1}{ }_{i q}=\min \left\{\left|\overline{\mathrm{C} P_{\mathrm{i}} C P_{q+1}}\right|,\left|\overline{\mathrm{C} P_{\mathrm{i}+1} C P_{q}}\right|\right\}$.

Concentricity measure: the concentricity measure between $E A S_{i}$ and $E A S_{q}$ is taken as the distance between their identified centres: $D_{i q}^{2}=\left|a_{i} a_{q}\right|$

Completeness measure: almost complete arcs tend to aggregate with less complete ones. The completeness measure of $E A S_{i}$ is taken as $C_{i}=\left|\overline{P_{1} a}\right| / \mid \overline{P_{1} P_{2} \mid}$. For two aggregation candidates $E A S_{i}$ and $E A S_{4}$, the neural network receives $C_{i}$ and $C_{q}$.

Energy measure: the "energy" resulting from the aggregation between two $E A S$ must be inversely proportional to the probability of both $E A S$ belonging to the same elliptical object. This measure is taken as (ord $\left(D_{i q}^{1}\right)$ - returns the order of $D_{i q}^{1}$ in the ordered set $\left.\left\{D^{1}{ }_{i j}\right\}, j=1, \ldots, N\right)$ :

$$
E_{i q}=\left(D_{i q}^{1}+D_{i q}^{2}\right)\left(1+1 / C_{i}+1 / C_{q}\right) \ln \left(2+\operatorname{ord}\left(D_{i q}^{1}\right)\right)
$$

Curvature similarity measure: for arcs $E A S_{i}$ and $E A S_{q}$, this measure is taken as:

$$
\operatorname{Curv}_{i q}=\max \left(R_{i} / R_{q}, R_{q} / R_{i}\right)
$$

All of these measures are normalised in the $[0,1]$ interval prior to their input to the neural network. After the learning stage, it is considered that two $E A S\left(E A S_{i}\right.$ and $\left.E A S_{q}\right)$ belong to the same elliptical object if the neural network's output $M C_{i q}>0.5$.

\section{Aggregation Path Search with Geometrical-Based Decision Criteria}

Although the membership indications obtained from the neural network are very reliable, it may happen that small errors occur (generally less than $0.5 \%$ ). On the other hand, given a high valued set of $M C$ it is not possible to infer from it the aggregation order of the associated $E A S$. To solve this problem a path search method is applied which handles critical boundary situations through neighbourhood analysis and a voting strategy. Aggregation decisions in the path search method are taken on the basis of a set of geometrical-based decision criteria:

Aggregation point criterion: the aggregation between $E A S_{\text {, and }} E A S_{q}$ must preserve the contour's direction. Therefore, this operation may only be performed between points $\left\{C P_{i}, C P_{4+1}\right\}$ or $\left\{C P_{i+1}, C P_{4}\right\}$. The actual choice is taken upon the minimum value of measure $D_{14}^{1}$.

Centre position criterion: if two $E A S$ aggregate together, then it is seen that their estimated centres must lie on the same side of the line segment defined by the contact points obtained with the aggregation point criterion.

Reciprocity criterion: two $E A S$ aggregate together, if the aggregation choice is mutual.

Shared adjacency criterion: if $E A S_{i}$ and $E A S_{q}$ aggregate together with aggregation points $\left\{C P_{i}, C P_{q+1}\right\}$ or $\left\{C P_{1+1}, C P_{4}\right\}$, then it is seen that, respectively, $E A S_{1-1}$ and $E A S_{4+1}$ or $E A S_{1+1}$ and $E A S_{4-1}$ will also aggregate.

Proximity criterion: aggregation points of sequential $E A S$ which belong to the same object tend to be localised at smaller distances from each other than to contact points of other $E A S$. Therefore, the aggregation candidate to $E A S_{i}$ should be $E A S_{q}$ if: 


$$
q=\operatorname{ArgMin}_{z=1, \ldots, N \wedge z=i}\left(D_{i z}^{1}\right)
$$

The detailed algorithm for the path search method for each identified cluster is given below:

For a given cluster build a $N \times N$ matrix $A$ such that $A_{i j}=M C_{i j}, i, j=1, \ldots, N$

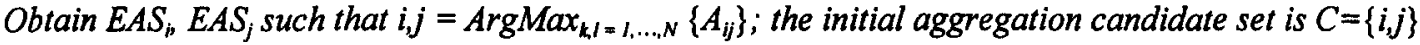
While (the absolute maximum in matrix $A$ is greater than 0.5 )

Extend set $C$ with all $E A S_{k}$ of the cluster such that $A_{l k}>0.5, l \in C, k=1, \ldots, N\{\{i, j\}$

If the number of $E A S$ identified in $C$ is 2 then

Apply the reciprocity, aggregation point and the centre position criteria

If all criteria are validated then Else

Both EAS are aggregated and the shared adjacency criterion is applied

The reciprocity criterion is checked for all $E A S$ in $C$

If all $E A S_{i} i \in C$, pass the reciprocity test with respect to all other $E A S_{j}, i \in C \wedge j \neq i$ then

For all $E A S_{i} i \in C$, the aggregation point, proximity and the centre position criteria are applied If both criteria are fulfilled then

The all $E A S_{i}, i \in C$, are aggregated and the shared adjacency criterion applied Else

$A$ voting strategy is followed, that is, each $E A S_{1}, i \in C$, votes for all $E A S_{j}, j \in C$, such that $M C_{i j}<0.5$. For each $E A S_{i}$ with less than $50 \%$ of the votes (all the others are discarded form $C$ ) the aggregation point criterion is applied:

If the candidate segment $E A S_{k}$ of $E A S_{i}$ has received 0 votes then

If the centre position criterion is verified for $E A S_{k}$ and $E A S_{i}$, the two segments are aggregated Else it is checked if $E A S_{k-1}$ and $E A S_{i+1}$ or $E A S_{k+1}$ and $E A S_{i-1}$ aggregate together. If this happens then because of the shared adjacency criterion segments $E A S_{k}$ of $E A S_{i}$ must also aggregate

Obtain the new $E A S_{i}, E A S_{j}$ for the initial set $C$ such that $i, j=\operatorname{ArgMax}_{k, 1=1, \ldots, N}\left\{A_{i j}\right\}$

\section{CONCLUSIONS}

In this paper a new approach to elliptical object localisation is introduced and applied to a lime granule inspection system. The method has proven to be robust, namely, it was able to correct several noisy information introduced in the neural network's output during the test procedure. Given the noise rejection ability of the path search method and the reduced membership degree error obtained by the neural network, these results may suggest the application of this method to other problems of different areas.

\section{REFERENCES}

Brink, A. D. and Pendock, N. E. (1996). Minimum cross-entropy threshold selection. Pattern Recognition, 29, 179-188.

Carvalho, P., Costa, N., Ribeiro, B. and Dourado, A. (1997). Lime quality inspection using a neural network and geometrical-based decision criteria. Proceedings of $12^{\text {th }}$ Int. Conf. on Systems Engineering, Coventry, 94-103.

Esplid, R. and Jonassen, I. (1991). A comparison of splitting methods for the identification of corner points. Pattern Recognition Letters. 12.

Jacobs, R. A. (1988). Increased rates of convergence through learning rate adaptation. Neural Networks. 1, 295-307.

Kim, C. and Rosenfeld, A. (1991). Digital straight lines and convexity of digital regions. IEEE Trans. On Pattern Analysis and Machine Vision.17.

Landau, U. M. (1987). Estimation of circular arc centre and its radius. Computer Vision, Graphics and Image Processing. 38, 317-326. 